PROCEEDINGS OF THE

AMERICAN MATHEMATICAL SOCIETY

Volume 138, Number 7, July 2010, Pages 2349-2364

S 0002-9939(10)10294-9

Article electronically published on March 10, 2010

\title{
GENERALIZATIONS OF GERSHGORIN DISKS AND POLYNOMIAL ZEROS
}

\author{
A. MELMAN
}

(Communicated by Walter Van Assche)

\begin{abstract}
We derive inclusion regions for the eigenvalues of a general complex matrix that are generalizations of Gershgorin disks, along with nonsingularity conditions. We then apply these results to the location of zeros of polynomials.
\end{abstract}

\section{INTRODUCTION}

A well-known inclusion region for eigenvalues of matrices is the Gershgorin set, composed of a union of disks. This result is stated in the following theorem, where the deleted row sum $R_{i}^{\prime}$ of a complex $n \times n$ matrix $A$ with elements $a_{i j}$ is defined as:

$$
R_{i}^{\prime}=\sum_{\substack{j=1 \\ j \neq i}}^{n}\left|a_{i j}\right| .
$$

The dependence of $R_{i}^{\prime}$ on $A$ was left out of the notation to avoid clutter, and similar notation will be used throughout this paper.

Theorem 1.1 (Gershgorin, [17]). All the eigenvalues of the $n \times n$ complex matrix $A$ are located in the union of the $n$ disks

$$
\bigcup_{i=1}^{n} \Gamma_{i}^{R} \equiv \Gamma^{R},
$$

where

$$
\Gamma_{i}^{R}=\left\{z \in \mathbb{C}:\left|z-a_{i i}\right| \leq R_{i}^{\prime}\right\} .
$$

The following result, due to A. Brauer, is also well-known.

Theorem 1.2 (Brauer, [3]). All the eigenvalues of the $n \times n$ complex matrix $A$ are located in the union of the $\left(\begin{array}{l}n \\ 2\end{array}\right)$ sets

$$
\bigcup_{\substack{i, j=1 \\ i<j}}^{n} B_{i j}^{R} \equiv B^{R},
$$

where

$$
B_{i j}^{R}=\left\{z \in \mathbb{C}|| z-a_{i i}|| z-a_{j j} \mid \leq R_{i}^{\prime} R_{j}^{\prime}\right\} \quad(i \neq j) .
$$

Received by the editors June 11, 2009, and, in revised form, October 25, 2009, and November 16, 2009.

2010 Mathematics Subject Classification. Primary 15A18, 12D10.

(C)2010 American Mathematical Society Reverts to public domain 28 years from publication 
The Brauer set $B^{R}$ is contained in the Gershgorin set $\Gamma^{R}$, and the boundaries of the $B_{i j}^{R}$ sets are quartic curves, called ovals of Cassini ([24, p. 153]).

The above results also have a column version since the spectra of $A$ and $A^{T}$ are identical.

There exist more complicated spectral inclusion sets, as in, e.g., [8, 9, , 10, 23, [30], and [33. A good survey of such sets can be found in [35, along with more advanced results, and for an introduction we refer to [19, Chapter 6].

We will derive sets that can be seen as generalizations of the Gershgorin disks, providing tighter bounds on the eigenvalues. They were inspired by the more restricted results in 29] for matrices with special symmetries. Although more complicated than disks, these sets can be very useful in special cases such as for sparse or structured matrices. The bulk of this paper is devoted to one such special case, namely to the location of the zeros of a polynomial, which are the eigenvalues of its (sparse) companion matrix, resulting in bounds on the moduli of the zeros. Our bounds are more complicated than similar bounds based on the Gershgorin set, but they require the same $\mathcal{O}(n)$ computational effort, where $n$ is the order of the polynomial. We note that this complexity refers to the computation of the aforementioned bounds, and not to the computation of the boundaries of the inclusion sets, which is more involved.

Bounds are, of course, no substitute for the accurate computation of polynomial zeros should that be required. However, they can be used to start a zero-finding method or in situations where it is sufficient to verify that the zeros lie in or are bounded away from a specific region.

The literature on the computation of polynomial zeros and bounds on such zeros is extensive, and we refer to [1, 44, [5], [6, 11, [12, 13, [15, 16, [20, 21, [22], 25], 26], 27, 28], 31, 32], 34, 37], 38], and the references therein, to name but a few. Most of these take a linear algebra approach, but some do not. There also exist several classical methods for the computation of polynomial zeros such as, e.g., the Laguerre and Durand-Kerner methods, and variations thereof. In addition, specialized methods were developed for special polynomials.

The organization of the paper is as follows. In Section 2 we derive our eigenvalue inclusion regions, which are then applied to computing bounds on the moduli of polynomial zeros in Section 3. Some examples are provided in Section 4.

\section{INCLUSION SETS}

Before we continue, we define for a matrix $A \in \mathbb{C}^{n \times n}$ :

$$
R_{i j}^{\prime \prime}=\sum_{\substack{k=1 \\ k \neq i, j}}^{n}\left|a_{i k}\right|=R_{i}^{\prime}-\left|a_{i j}\right| .
$$

We also define the set $J_{i}=\{1,2, \ldots, n\} \backslash\{i\}$.

Although we will concentrate on the rows, analogous column versions of our results can easily be obtained by considering $A^{T}$ instead of $A$.

The next theorem presents our generalized Gershgorin spectral inclusion sets. 
Theorem 2.1. All the eigenvalues of the $n \times n$ complex matrix $A$ are located in the union of the following $n$ sets:

$$
\bigcup_{i=1}^{n}\left\{\bigcap_{j \in S_{i}} \Omega_{i j}^{R}\right\}
$$

where $S_{i}$ is any nonempty subset of $J_{i}$ and

$$
\Omega_{i j}^{R}=\left\{z \in \mathbb{C}:\left|\left(z-a_{i i}\right)\left(z-a_{j j}\right)-a_{i j} a_{j i}\right| \leq\left|z-a_{j j}\right| R_{i j}^{\prime \prime}+\left|a_{i j}\right| R_{j i}^{\prime \prime}\right\} \quad(i \neq j) .
$$

Proof. The proof is similar to that of Gershgorin's theorem ([19, pp. 344-345]). Let $\lambda$ be an eigenvalue of $A$ with corresponding eigenvector $x$, i.e., $A x=\lambda x$. Since $x$ is an eigenvector, it has at least one nonzero component. Define $x_{\rho}$ as a component of $x$ with the largest absolute value, so that $\left|x_{\rho}\right| \geq\left|x_{j}\right|$ for all $j=1,2, \ldots, n$ and $x_{\rho} \neq 0$.

For any $\mu \neq \rho$ we then have

$$
\begin{aligned}
\lambda x_{\rho} & =\sum_{\substack{j=1 \\
j \neq \rho, \mu}}^{n} a_{\rho j} x_{j}+a_{\rho \rho} x_{\rho}+a_{\rho \mu} x_{\mu}, \\
\lambda x_{\mu} & =\sum_{\substack{j=1 \\
j \neq \rho, \mu}}^{n} a_{\mu j} x_{j}+a_{\mu \rho} x_{\rho}+a_{\mu \mu} x_{\mu},
\end{aligned}
$$

which is equivalent to

$$
\begin{aligned}
\left(\lambda-a_{\rho \rho}\right) x_{\rho}-a_{\rho \mu} x_{\mu} & =\sum_{\substack{j=1 \\
j \neq \rho, \mu}}^{n} a_{\rho j} x_{j}, \\
-a_{\mu \rho} x_{\rho}+\left(\lambda-a_{\mu \mu}\right) x_{\mu} & =\sum_{\substack{j=1 \\
j \neq \rho, \mu}}^{n} a_{\mu j} x_{j} .
\end{aligned}
$$

Solving for $x_{\rho}$ we obtain

$$
\left(\left(\lambda-a_{\rho \rho}\right)\left(\lambda-a_{\mu \mu}\right)-a_{\rho \mu} a_{\mu \rho}\right) x_{\rho}=\left(\lambda-a_{\mu \mu}\right) \sum_{\substack{j=1 \\ j \neq \rho, \mu}}^{n} a_{\rho j} x_{j}+a_{\rho \mu} \sum_{\substack{j=1 \\ j \neq \rho, \mu}}^{n} a_{\mu j} x_{j} .
$$

Taking the absolute value on both sides of the equation and using the triangle inequality yields

$$
\left|\left(\lambda-a_{\rho \rho}\right)\left(\lambda-a_{\mu \mu}\right)-a_{\rho \mu} a_{\mu \rho}\right|\left|x_{\rho}\right| \leq\left|\lambda-a_{\mu \mu}\right| \sum_{\substack{j=1 \\ j \neq \rho, \mu}}^{n}\left|a_{\rho j}\right|\left|x_{j}\right|+\left|a_{\rho \mu}\right| \sum_{\substack{j=1 \\ j \neq \rho, \mu}}^{n}\left|a_{\mu j}\right|\left|x_{j}\right| .
$$

Since $x_{\rho} \neq 0$ and $\left|x_{\rho}\right| \geq\left|x_{j}\right|$ for all $j$, we can divide through by $\left|x_{\rho}\right|$ to obtain

$$
\left|\left(\lambda-a_{\rho \rho}\right)\left(\lambda-a_{\mu \mu}\right)-a_{\rho \mu} a_{\mu \rho}\right| \leq\left|\lambda-a_{\mu \mu}\right| R_{\rho \mu}^{\prime \prime}+\left|a_{\rho \mu}\right| R_{\mu \rho}^{\prime \prime} .
$$

For each $\mu \neq \rho$ we have such an inequality, which means that the eigenvalue must lie in the intersection of the sets defined by these inequalities. One has the freedom to choose only one $\mu$ (in which case there are no intersections) or more than one. On the other hand, we do not know which $\rho$ corresponds to a given eigenvalue and can therefore only say that any eigenvalue must lie in the union of all $n$ sets determined by the corresponding $\rho$ 's. This concludes the proof. 
In general, the sets $\Omega_{i j}^{R}$ are bounded by algebraic curves of degree 8 that are somewhat reminiscent of the Cassini ovals which appear in the Brauer inclusion set. Special cases of the sets $\Omega_{i j}^{R}$ include singletons, disks and sets bounded by ovals of Cassini. Some typical general examples can be seen in Figure 1, The rightmost set consists of two separate loops.
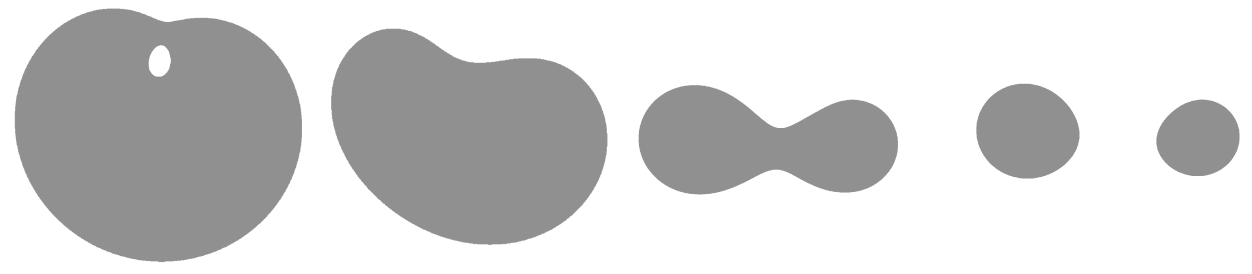

Figure 1. Examples of the $\Omega_{i j}^{R}$ sets.

In the next theorem we show that the $\Omega_{i j}^{R}$ sets are contained in Gershgorin disks, so that the eigenvalue inclusion set in (2.1) always lies in the Gershgorin set. Nevertheless, it can range from not very different to dramatically different from the Gershgorin set. Our sets are, in general, not contained in the Brauer set, although they frequently are in practice. In Figure 2 we have provided a few typical examples for randomly generated matrices of dimension 8. The Gershgorin sets are shaded in intermediate grey, the Brauer sets in light grey, and the sets from Theorem 2.1 with $S_{i}=J_{i}$ for all $i$ in dark grey. The eigenvalues are indicated by the white dots.
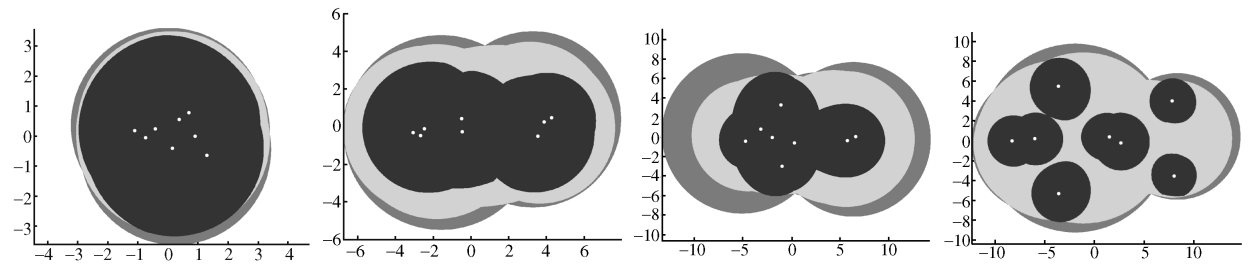

Figure 2. Comparison of Gershgorin, Brauer, and generalized

Gershgorin sets.

Theorem 2.2. The sets $\Omega_{i j}^{R}$ satisfy

$$
\Omega_{i j}^{R} \subseteq \Gamma_{i}^{R} \cup \Gamma_{j}^{R} \quad \text { for all } i \neq j .
$$

The union of intersections of such sets is therefore contained in the Gershgorin set $\Gamma^{R}$.

Proof. Assume that $z \in \Omega_{i j}^{R}$ for some $i, j \in\{1, \ldots, n\}$ and $i \neq j$. Then $z \in \Gamma_{i}^{R}$ or $z \notin \Gamma_{i}^{R}$. If $z \in \Gamma_{i}^{R}$, there is nothing to prove. Assume therefore that $z \notin \Gamma_{i}^{R}$. Since $z \in \Omega_{i j}^{R}$, we have

$\left|z-a_{j j}\right| R_{i j}^{\prime \prime}+\left|a_{i j}\right| R_{j i}^{\prime \prime} \geq\left|\left(z-a_{i i}\right)\left(z-a_{j j}\right)-a_{i j} a_{j i}\right| \geq\left|z-a_{i i}\right|\left|z-a_{j j}\right|-\left|a_{i j}\right|\left|a_{j i}\right|$.

Because $R_{i j}^{\prime \prime}=R_{i}^{\prime}-\left|a_{i j}\right|$ and $R_{j i}^{\prime \prime}=R_{j}^{\prime}-\left|a_{j i}\right|$, and because $\left|z-a_{i i}\right|>R_{i}^{\prime}$, we have from (2.2) that

$$
\left|z-a_{j j}\right|\left(R_{i}^{\prime}-\left|a_{i j}\right|\right)+\left|a_{i j}\right|\left(R_{j}^{\prime}-\left|a_{j i}\right|\right) \geq R_{i}^{\prime}\left|z-a_{j j}\right|-\left|a_{i j}\right|\left|a_{j i}\right|,
$$


which implies that

$$
\left|a_{i j}\right| R_{j}^{\prime} \geq\left|a_{i j}\right|\left|z-a_{j j}\right| .
$$

If $a_{i j} \neq 0$, then this inequality means that $z \in \Gamma_{j}^{R}$. In other words, if $z$ is not in $\Gamma_{i}^{R}$, then it must be in $\Gamma_{j}^{R}$. If $a_{i j}=0$, then it is easy to see that $\Omega_{i j}^{R}=\Gamma_{i}^{R} \cup\left\{a_{j j}\right\} \subseteq$ $\Gamma_{i}^{R} \cup \Gamma_{j}^{R}$. This completes the proof.

Just as nonsingularity conditions lead to eigenvalue inclusion sets (a central theme in [35]), an eigenvalue inclusion set for a matrix naturally leads to nonsingularity conditions for that matrix. The following theorem presents such conditions.

Theorem 2.3. Let $A \in \mathbb{C}^{n \times n}$. Then $A$ is invertible if for each $i=1,2, \ldots, n$, there exists a $j \neq i$ such that

$$
\left|a_{i i} a_{j j}-a_{i j} a_{j i}\right|>\left|a_{j j}\right| R_{i j}^{\prime \prime}+\left|a_{i j}\right| R_{j i}^{\prime \prime} .
$$

Proof. The proof follows immediately by requiring that zero not lie in the spectral inclusion set described by (2.1) in Theorem 2.1 with $S_{i}=J_{i}$ for all $i$.

We note that, as is the case for the Gershgorin set, our results can be improved by using a suitable similar matrix $S^{-1} A S$ instead of $A$, which has the same eigenvalues.

\section{Polynomial zeros}

Eigenvalue inclusion sets can be used to estimate zeros of polynomials by applying them to the polynomial's companion matrix (see, e.g., [19, p. 146]), whose eigenvalues are the zeros of the polynomial. For a monic polynomial $p(z)=$ $z^{n}+\alpha_{n-1} z^{n-1}+\cdots+\alpha_{1} z+\alpha_{0}$ with complex coefficients and with $\alpha_{0} \neq 0$, the companion matrix is given by

$$
\left(\begin{array}{ccccc}
0 & 0 & \ldots & 0 & -\alpha_{0} \\
1 & 0 & \ldots & 0 & -\alpha_{1} \\
0 & 1 & \ldots & 0 & -\alpha_{2} \\
\vdots & \vdots & \vdots & \vdots & \vdots \\
0 & 0 & \ldots & 1 & -\alpha_{n-1}
\end{array}\right) .
$$

We concentrate on this frequently used companion matrix, but there exist other companion matrices that could also be used, as in, e.g., [2, [6], 7], 14, [18, 25, [26, 27], or 36. In the following theorem we derive an inclusion set for polynomial zeros, based on Theorem 2.1 .

Theorem 3.1. All the zeros of the polynomial $p(z)=z^{n}+\alpha_{n-1} z^{n-1}+\cdots+\alpha_{1} z+\alpha_{0}$ with complex coefficients and with $\alpha_{0} \neq 0$ lie in the union of the $n$ sets

$$
\bigcup_{i=1}^{n} \Phi_{i} \equiv \Phi,
$$

where

$$
\begin{aligned}
& \Phi_{1}=\left\{z \in \mathbb{C}:|z| \leq\left|\alpha_{0}\right| \text { and }|z|\left|z+\alpha_{n-1}\right| \leq\left|\alpha_{0}\right|\right\} \\
& \Phi_{i}=\left\{z \in \mathbb{C}:|z| \leq \mu_{i} \text { and }\left|z\left(z+\alpha_{n-1}\right)\right| \leq\left|z+\alpha_{n-1}\right|+\left|\alpha_{i-1}\right|\right\} \quad(2 \leq i \leq n-2), \\
& \Phi_{n-1}=\left\{z \in \mathbb{C}:|z| \leq \mu_{n-1} \text { and }\left|z\left(z+\alpha_{n-1}\right)+\alpha_{n-2}\right| \leq\left|z+\alpha_{n-1}\right|\right\} \\
& \Phi_{n}=\left\{z \in \mathbb{C}:\left|z+\alpha_{n-1}\right| \leq 1 \text { and }\left|z\left(z+\alpha_{n-1}\right)+\alpha_{n-2}\right| \leq 1\right\}
\end{aligned}
$$


and

$$
\begin{aligned}
& \mu_{1}=\left|\alpha_{0}\right|, \mu_{2}=\min \left\{1+\left|\alpha_{1}\right|, \frac{1}{2}\left(\left|\alpha_{1}\right|+\sqrt{\left|\alpha_{1}\right|^{2}+4\left|\alpha_{0}\right|}\right)\right\}, \\
& \mu_{i}=\min \left\{1+\left|\alpha_{i-1}\right|, \frac{1}{2}\left(\left|\alpha_{i-1}\right|+\sqrt{\left|\alpha_{i-1}\right|^{2}+4\left(1+\left|\alpha_{i-2}\right|\right.}\right)\right\} \quad(i=3, \ldots, n-1) .
\end{aligned}
$$

Proof. Applying Theorem 2.1 with $S_{i}=J_{i}$ for all $i=1,2, \ldots, n$ to the companion matrix of $p(z)$ results in the following $\Omega_{i, j}^{R}$ sets, stated in their raw unsimplified form.

For $i=1$ :

$$
\begin{aligned}
& \Omega_{1, j}^{R}=\left\{z \in \mathbb{C}:|z|^{2} \leq|z|\left|\alpha_{0}\right|\right\} \quad(2 \leq j \leq n-1) \\
& \Omega_{1, n}^{R}=\left\{z \in \mathbb{C}:|z|\left|z+\alpha_{n-1}\right| \leq\left|\alpha_{0}\right|\right\}
\end{aligned}
$$

For $i=2$ :

$$
\begin{aligned}
& \Omega_{2, j}^{R}=\left\{z \in \mathbb{C}:|z|^{2} \leq|z|\left(1+\left|\alpha_{1}\right|\right)\right\} \quad(3 \leq j \leq n-1) \\
& \Omega_{2,1}^{R}=\left\{z \in \mathbb{C}:|z|^{2} \leq|z|\left|\alpha_{1}\right|+\left|\alpha_{0}\right|\right\} \\
& \Omega_{2, n}^{R}=\left\{z \in \mathbb{C}:\left|z\left(z+\alpha_{n-1}\right)\right| \leq\left|z+\alpha_{n-1}\right|+\left|\alpha_{1}\right|\right\}
\end{aligned}
$$

For $3 \leq i \leq n-2$ :

$$
\begin{aligned}
& \Omega_{i, j}^{R}=\left\{z \in \mathbb{C}:|z|^{2} \leq|z|\left(1+\left|\alpha_{i-1}\right|\right)\right\} \quad(1 \leq j \leq i-2 \text { or } i+1 \leq j \leq n-1) \\
& \Omega_{i, i-1}^{R}=\left\{z \in \mathbb{C}:|z|^{2} \leq|z|\left|\alpha_{i-1}\right|+1+\left|\alpha_{i-2}\right|\right\} \\
& \Omega_{i, n}^{R}=\left\{z \in \mathbb{C}:\left|z\left(z+\alpha_{n-1}\right)\right| \leq\left|z+\alpha_{n-1}\right|+\left|\alpha_{i-1}\right|\right\}
\end{aligned}
$$

For $i=n-1$ :

$$
\begin{aligned}
\Omega_{n-1, j}^{R} & =\left\{z \in \mathbb{C}:|z|^{2} \leq|z|\left(1+\left|\alpha_{n-2}\right|\right)\right\} \quad(1 \leq j \leq n-3), \\
\Omega_{n-1, n-2}^{R} & =\left\{z \in \mathbb{C}:|z|^{2} \leq|z|\left|\alpha_{n-2}\right|+1+\left|\alpha_{n-3}\right|\right\} \\
\Omega_{n-1, n}^{R} & =\left\{z \in \mathbb{C}:\left|z\left(z+\alpha_{n-1}\right)+\alpha_{n-2}\right| \leq\left|z+\alpha_{n-1}\right|\right\} .
\end{aligned}
$$

For $i=n$ :

$$
\begin{aligned}
\Omega_{n, j}^{R} & =\left\{z \in \mathbb{C}:\left|z\left(z+\alpha_{n-1}\right)\right| \leq|z|\right\} \quad(1 \leq j \leq n-2), \\
\Omega_{n, n-1}^{R} & =\left\{z \in \mathbb{C}:\left|z\left(z+\alpha_{n-1}\right)+\alpha_{n-2}\right| \leq 1\right\} .
\end{aligned}
$$

We first consider $i=2, \ldots, n-1$. In this case, the set appearing in the definition of $\Phi_{i}$ of the form $\left\{z \in \mathbb{C}:|z|^{2} \leq \alpha|z|+\beta\right\}$, where $\alpha$ and $\beta$ are real and nonnegative, is equivalent to

$$
\left\{z \in \mathbb{C}:|z|^{2}-\alpha|z|-\beta \leq 0\right\} .
$$

Such a set is easily seen to be a disk of the form

$$
\left\{z \in \mathbb{C}:|z| \leq \frac{1}{2}\left(\alpha+\sqrt{\alpha^{2}+4 \beta}\right)\right\} .
$$

Furthermore, the set $\Omega_{i, j}^{R}(j \neq i-1, i, n)$ is the same as $\left\{z \in \mathbb{C}:|z| \leq 1+\left|\alpha_{i-1}\right|\right\}$ since the latter includes $\{0\}$.

The situation for $i=1$ is analogous. For $i=n-1, n$ we can exclude $z=0$ and divide out $|z|$ in the definition of $\Omega_{n-1, j}^{R}(1 \leq j \leq n-3)$ and $\Omega_{n, j}^{R}(1 \leq j \leq n-2)$ because $\{0\}$ is included in all the other sets $\Phi_{i}(i \neq n-1, n)$ and we are taking their union. With the definition of $\mu_{i}(i=1, \ldots, n-1)$, this concludes the proof. 
Examples. We illustrate the inclusion sets in the previous theorem with a few examples. The polynomial coefficients in these examples were randomly generated and no special conditions were imposed. The inclusion sets for the following polynomials can be seen in Figure 3

$$
\begin{gathered}
p_{1}(z)=z^{8}+2 z^{7}-4 z^{6}+i z^{5}+(1-3 i) z^{4}-(5+2 i) z^{3}-(4-6 i) z^{2} \\
-(2+4 i) z+4+2 i, \\
p_{2}(z)=z^{10}-(4+8 i) z^{9}+3 i z^{8}-(5+3 i) z^{7}+(1-8 i) z^{6}+(7+i) z^{5} \\
\quad-(4-6 i) z^{4}-(9-3 i) z^{3}-(6-9 i) z^{2}-(4-9 i) z+1-9 i, \\
p_{3}(z)=z^{8}+3 z^{6}-2 z^{4}+2 z^{3}+z^{2}-z+2, \\
p_{4}(z)=z^{10}-7 z^{9}-3 z^{8}+4 z^{7}-6 z^{6}-9 z^{5}+4 z^{4}-9 z^{3}+2 z^{2}+10 z+5 .
\end{gathered}
$$

The generalized Gershgorin sets (dark grey) are contained well inside the Brauer sets (light grey) and the Gershgorin sets (intermediate grey). The zeros are indicated by the white dots.
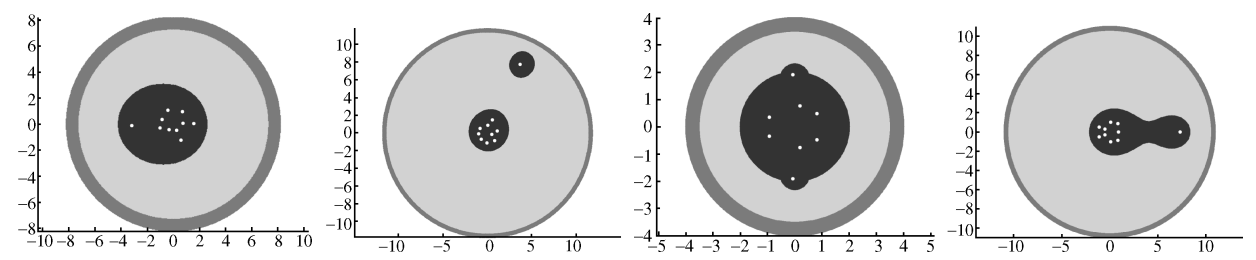

Figure 3. Comparison of inclusion regions for the zeros of $p_{1}(z)$, $p_{2}(z), p_{3}(z)$, and $p_{4}(z)$.

Let us now have a closer look at the sets appearing in $\Phi$. Many of them are just disks and need no explanation. However, some are a little more complicated.

We start with $\Phi_{1}$, which contains a set of the form

$$
\{z \in \mathbb{C}:|z-a||z-b| \leq \alpha\}
$$

where $a$ and $b$ are complex numbers, and $\alpha$ is real and nonnegative.

For positive $\alpha$, and for $a \neq b$, the boundary of this set is an oval of Cassini with foci $a$ and $b$, and when $\alpha=0$ the set reduces to the two points $\{a\}$ and $\{b\}$. When $a=b$, the boundary becomes a circle for $\alpha>0$ and a point for $\alpha=0$.

An oval of Cassini also appears in $\Phi_{n}$ as the boundary of a set, namely

$$
\left\{z \in \mathbb{C}:\left|z\left(z+\alpha_{n-1}\right)+\alpha_{n-2}\right|=1\right\},
$$

which can be seen by writing the equation in (3.1) as

$$
\left|\left(z-\frac{1}{2}\left(-\alpha_{n-1}-\sqrt{\alpha_{n-1}^{2}-4 \alpha_{n-2}}\right)\right)\right|\left|\left(z-\frac{1}{2}\left(-\alpha_{n-1}+\sqrt{\alpha_{n-1}^{2}-4 \alpha_{n-2}}\right)\right)\right|=1 .
$$

A slightly more complicated set turns up in $\Phi_{i}(i=2, \ldots, n-2)$, namely

$$
\left\{z \in \mathbb{C}:\left|z\left(z+\alpha_{n-1}\right)\right| \leq\left|z+\alpha_{n-1}\right|+\left|\alpha_{i-1}\right|\right\} .
$$

Its boundary is a curve of degree eight, and using Cartesian or polar coordinates to obtain its basic properties would be quite complicated. The following lemma uses geometric arguments instead. 
Lemma 3.2. The curve defined by

$$
\{z \in \mathbb{C}:|z-a||z-b|=\alpha|z-a|+\beta\},
$$

where $a$ and $b$ are distinct complex numbers and $\alpha \geq 0$ and $\beta>0$, is symmetric with respect to the line through $a$ and $b$ and intersects that line at a distance of $R_{\max }$ from $a$ in the direction of $b$ and $R_{\text {min }}$ from $a$ in the opposite direction, away from $b$, where

$$
\begin{aligned}
& R_{\text {min }}=\frac{1}{2}\left(\alpha-|a-b|+\sqrt{(|a-b|-\alpha)^{2}+4 \beta}\right), \\
& R_{\text {max }}=\frac{1}{2}\left(\alpha+|a-b|+\sqrt{(|a-b|+\alpha)^{2}+4 \beta}\right) .
\end{aligned}
$$

If $|a-b| \geq \alpha+2 \sqrt{\beta}$, then there are two additional points of intersection, which coincide when $|a-b|=\alpha+2 \sqrt{\beta}$, namely at a distance of $Q_{\min }$ and $Q_{\max }$ from a in the direction of $b$, where

$$
\begin{aligned}
Q_{\min } & =\frac{1}{2}\left(|a-b|-\alpha-\sqrt{(|a-b|-\alpha)^{2}-4 \beta}\right), \\
Q_{\max } & =\frac{1}{2}\left(|a-b|-\alpha+\sqrt{(|a-b|-\alpha)^{2}-4 \beta}\right) .
\end{aligned}
$$

If $\beta=0$, the curve becomes the union of a circle and a point when $\alpha \neq 0$, or it degenerates into two points when $\alpha=0$.

For $a=b$, the curve becomes a circle when $\beta \neq 0$ and when $\beta=0$, it becomes the union of a circle and a point, or just a point, depending on whether $\alpha$ is nonzero or not, respectively.

Proof. First, assume that $a \neq b$ and $\beta>0$. Then, since $z=a$ does not satisfy (3.2), we can equivalently write the equation as

$$
|z-b|=\alpha+\frac{\beta}{|z-a|} .
$$

This means that $z$ is a point that lies on a circle with a radius $R$ and center $a$ (Circle 1) and also on a circle with radius $\alpha+\beta / R$ and center $b$ (Circle 2). But $R$ cannot be just any positive number. As $R \rightarrow 0^{+}$, the two circles clearly cannot intersect and Circle 1 will be entirely contained in Circle 2. As $R$ increases, Circle 1 grows while Circle 2 shrinks. At some point the two circles will touch. This happens when $R+|a-b|=\alpha+\beta / R$. Discarding the negative solution of the resulting quadratic equation for $R$, we thus obtain that the minimum radius $R$ is given by expression (3.3) in the statement of the lemma. There can be no intersections for $R<R_{\text {min }}$.

As $R$ increases from $R_{\min }$, the two circles now intersect in two points, symmetric with respect to the line through the points $a$ and $b$. On the other hand, $R$ can be so large that Circle 2 is entirely contained in Circle 1 and touches it at just one point. This happens when $|a-b|+\alpha+\beta / R=R$, so that we obtain expression (3.4) in the statement of the lemma. As $R$ decreases from $R_{\max }$, the two circles now intersect in two points, symmetric with respect to the line through the points $a$ and $b$. There can be no intersections for $R>R_{\max }$.

It could also happen that, as $R$ increases from $R_{\text {min }}$, Circle 2 shrinks so fast that there comes a point at which it no longer intersects Circle 1 . If that is the case, then the value of $R$ for which this happens will be denoted by $Q_{\min }$. When $R$ continues to increase after this point, Circle 2 will inevitably catch up with Circle 1 again. 
The value of $R$ at which that happens will be denoted by $Q_{\max }$. Each of these events will occur only once or none will occur at all because at both of these events we must have that $R+\alpha+\beta / R=|a-b|$, and this particular quadratic equation can only have two positive (possibly equal) solutions, two negative solutions, or two imaginary solutions. Clearly, the positive solutions are the only ones of interest.

An easy computation shows that the two solutions $Q_{\min }$ and $Q_{\max }$ are given by expressions (3.5) and (3.6) in the statement of the theorem. Obviously, this situation only arises when $|a-b| \geq \alpha+2 \sqrt{\beta}$. When the inequality is strict, the curve has two disjoint loops, which are contained in the union of two disks: one with center $a$ and radius $Q_{\min }$ and one with center $b$ and radius $\alpha+\beta / Q_{\max }=|a-b|-Q_{\max }$.

Figures 4 and 5 show the evolution of the intersections of Circle 1 (solid line), centered at $a$ (upper right), and Circle 2 (dotted line), centered at $b$ (lower left), as $R$ goes from $R_{\min }$ to $R_{\max }$ through $Q_{\min }$ and $Q_{\max }$, i.e., in the situation that we have two loops.

The proof for the cases $\beta=0$ and $a=b$ mentioned at the end of the statement of the lemma are straightforward.

The curve defined in (3.2) is an oval of Cassini when $\alpha=0$. Other properties of this curve such as, e.g., minimum and maximum distances from the line through $a$ and $b$, can be derived using the same techniques as in Lemma 3.2. We do not need these properties here and will omit them for brevity's sake.

Using the same notation as in Lemma 3.2, we note that the set defined by

$$
\{z \in \mathbb{C}:|z-a||z-b| \leq \alpha|z-a|+\beta\}
$$

contains the points $a$ and $b$ and the area enclosed by the loop containing them. The points $a$ and $b$ can be considered the foci of the boundary curve.

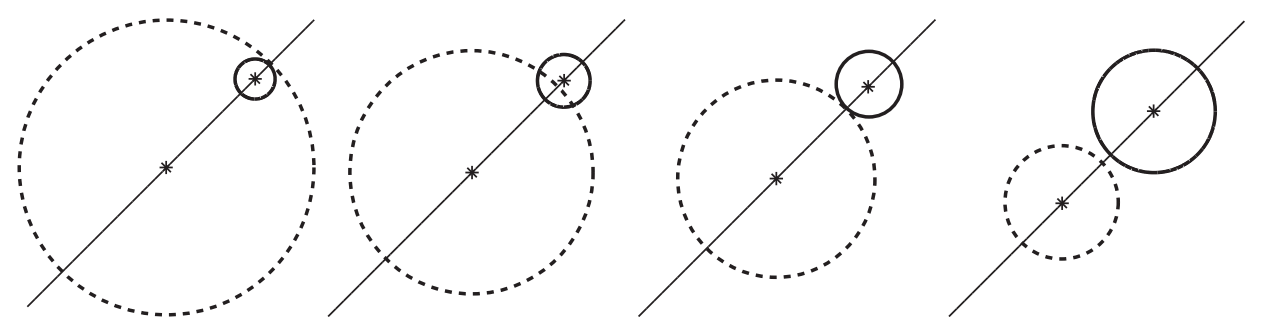

FIguRE 4. Evolution as $R$ increases from $R_{\min }$ (left) to a value between $Q_{\min }$ and $Q_{\max }$ (right).
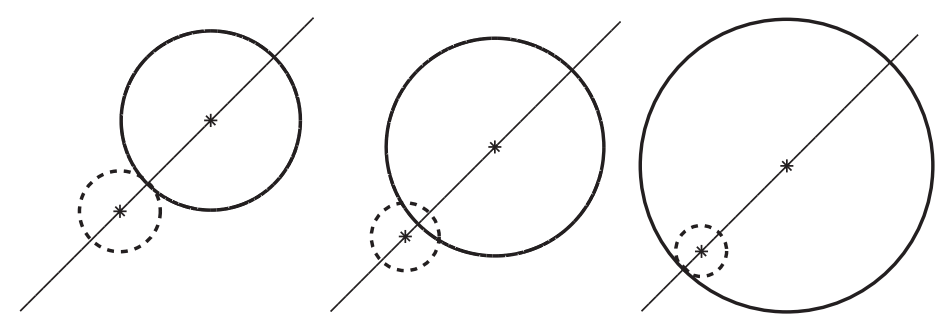

Figure 5. Evolution as $R$ increases from $Q_{\max }$ (left) to $R_{\max }$ (right).

The following theorem makes use of the inclusion sets $\Phi_{i}$ to derive bounds on the modulus of the zeros of a polynomial. 
Theorem 3.3. Given the polynomial $p(z)=z^{n}+\alpha_{n-1} z^{n-1}+\cdots+\alpha_{1} z+\alpha_{0}$ with complex coefficients and with $\alpha_{0} \neq 0$, defining its corresponding zero inclusion sets $\Phi_{i}$ as in Theorem 3.1, and defining $\beta_{i}, \mu_{i}, q_{i}^{+}$, and $q_{i}^{-}$for $i=1, \ldots, n$, and $r_{i}$ for $i=1, \ldots, n-1$ as

$$
\begin{aligned}
& \beta_{1}=2 \sqrt{\left|\alpha_{0}\right|}, \beta_{n}=2 \sqrt{1+\left|\alpha_{n-2}\right|}, \beta_{i}=1+2 \sqrt{\left|\alpha_{i-1}\right|}(i \neq 1, n), \\
& \mu_{1}=\left|\alpha_{0}\right|, \mu_{2}=\min \left\{1+\left|\alpha_{1}\right|, \frac{1}{2}\left(\left|\alpha_{1}\right|+\sqrt{\left|\alpha_{1}\right|^{2}+4\left|\alpha_{0}\right|}\right)\right\}, \\
& \mu_{i}=\min \left\{1+\left|\alpha_{i-1}\right|, \frac{1}{2}\left(\left|\alpha_{i-1}\right|+\sqrt{\left|\alpha_{i-1}\right|^{2}+4\left(1+\left|\alpha_{i-2}\right|\right)}\right)\right\} \quad(i \neq 1,2), \\
& r_{1}=\frac{1}{2}\left(\left|\alpha_{n-1}\right|+\sqrt{\left|\alpha_{n-1}\right|^{2}+4\left|\alpha_{0}\right|}\right), \\
& r_{i}=\frac{1}{2}\left(1+\left|\alpha_{n-1}\right|+\sqrt{\left(\left|\alpha_{n-1}\right|-1\right)^{2}+4\left|\alpha_{i-1}\right|}\right) \quad(i \neq 1), \\
& q_{1}^{-}=\frac{1}{2}\left(\left|\alpha_{n-1}\right|-\sqrt{\left|\alpha_{n-1}\right|^{2}-4\left|\alpha_{0}\right|}\right), \\
& q_{n}^{-}=\frac{1}{2}\left(\left|\alpha_{n-1}\right|-\sqrt{\left|\alpha_{n-1}\right|^{2}-4\left(1+\left|\alpha_{n-2}\right|\right)}\right), \\
& q_{i}^{-}=\frac{1}{2}\left(1+\left|\alpha_{n-1}\right|-\sqrt{\left(\left|\alpha_{n-1}\right|-1\right)^{2}-4\left|\alpha_{i-1}\right|}\right) \quad(i \neq 1, n), \\
& q_{1}^{+}=\frac{1}{2}\left(\left|\alpha_{n-1}\right|+\sqrt{\left|\alpha_{n-1}\right|^{2}-4\left|\alpha_{0}\right|}\right), \\
& q_{n}^{+}=\frac{1}{2}\left(\left|\alpha_{n-1}\right|+\sqrt{\left|\alpha_{n-1}\right|^{2}-4\left(1+\left|\alpha_{n-2}\right|\right)}\right), \\
& q_{i}^{+}=\frac{1}{2}\left(1+\left|\alpha_{n-1}\right|+\sqrt{\left(\left|\alpha_{n-1}\right|-1\right)^{2}-4\left|\alpha_{i-1}\right|}\right) \quad(i \neq 1, n),
\end{aligned}
$$

the following holds for $1 \leq i \leq n-1$ :

(1) If $\left|\alpha_{n-1}\right| \leq \beta_{i}$, then $\forall z \in \Phi_{i}: 0 \leq|z| \leq \min \left\{\mu_{i}, r_{i}\right\}$.

(2) If $\left|\alpha_{n-1}\right|>\beta_{i}$ and $\mu_{i} \geq q_{i}^{+}$, then $\forall z \in \Phi_{i}$ :

$$
0 \leq|z| \leq q_{i}^{-} \text {or } q_{i}^{+} \leq|z| \leq \min \left\{\mu_{i}, r_{i}\right\} .
$$

(3) If $\left|\alpha_{n-1}\right|>\beta_{i}$ and $\mu_{i}<q_{i}^{+}$, then $\forall z \in \Phi_{i}: 0 \leq|z| \leq \min \left\{\mu_{i}, q_{i}^{-}\right\}$. For $i=n$ the following holds:

(4) If $\left|\alpha_{n-1}\right| \leq \beta_{n}$, then $\forall z \in \Phi_{n}: \max \left\{0,\left|\alpha_{n-1}\right|-1\right\} \leq|z| \leq \mu_{n}$.

(5) If $\left|\alpha_{n-1}\right|>\beta_{n}$, then $\forall z \in \Phi_{n}: \max \left\{q_{n}^{+},\left|\alpha_{n-1}\right|-1\right\} \leq|z| \leq \mu_{n}$.

Proof. We start with $2 \leq i \leq n-2$. The sets $\Phi_{i}$ for $2 \leq i \leq n-2$ are given by $\Phi_{i}=\left\{z \in \mathbb{C}:|z| \leq \mu_{i}\right.$ and $\left.\left|z\left(z+\alpha_{n-1}\right)\right| \leq\left|z+\alpha_{n-1}\right|+\left|\alpha_{i-1}\right|\right\} \quad(2 \leq i \leq n-2)$.

Each of those sets is the intersection of a disk, centered at the origin, with a region, bounded by the curve

$$
\left\{z \in \mathbb{C}:\left|z\left(z+\alpha_{n-1}\right)\right|=\left|z+\alpha_{n-1}\right|+\left|\alpha_{i-1}\right|\right\} \quad(2 \leq i \leq n-2),
$$

i.e., a curve of the same form as in Lemma 3.2, with, in the notation of that lemma, $a=-\alpha_{n-1}, b=0, \alpha=1$, and $\beta=\left|\alpha_{i-1}\right|$.

If $\left|\alpha_{n-1}\right| \leq \beta_{i}=1+2 \sqrt{\left|\alpha_{i-1}\right|}$, the curve has only one loop. As was explained in Lemma 3.2, we can interpret the curve as the intersection points of two circles, one of which is, in this case, centered at the origin. Obviously, the modulus of any point on the curve is equal to the radius of the corresponding circle centered at the 
origin and passing through that point. Lemma 3.2 then implies that the maximum value for $|z|$ when $z$ lies on or inside the curve is given by $r_{i} \equiv\left|\alpha_{n-1}\right|+R_{\text {min }}$, where we used the same notation as in Lemma 3.2, and we have that

$$
r_{i}=\frac{1}{2}\left(1+\left|\alpha_{n-1}\right|+\sqrt{\left(\left|\alpha_{n-1}\right|-1\right)^{2}+4\left|\alpha_{i-1}\right|}\right) .
$$

We note that $|z|$ attains all values between 0 and its maximum value in $\Phi_{i}$ because there is only one loop. Since $\Phi_{i}$ is obtained by intersecting the region bounded by the aforementioned curve with a disk, centered at the origin and with radius $\mu_{i}$, statement (1) of the theorem for $2 \leq i \leq n-2$ follows immediately.

If $\left|\alpha_{n-1}\right|>\beta_{i}=1+2 \sqrt{\left|\alpha_{i-1}\right|}$, then the curve has two loops, which means that it intersects the line through the origin and $-\alpha_{n-1}$ in two additional places, which are at a distance of $q_{i}^{+} \equiv\left|\alpha_{n-1}\right|-Q_{\min }$ and $q_{i}^{-} \equiv\left|\alpha_{n-1}\right|-Q_{\max }$ from the origin, respectively. Clearly, $q_{i}^{-} \leq q_{i}^{+}$. We have once again used the same notation as in Lemma 3.2, and it is worth pointing out that here, as elsewhere in this proof, $Q_{\min }$ and $Q_{\max }$ are distances measured from $-\alpha_{n-1}$, whereas $q_{i}^{-}$and $q_{i}^{+}$are distances measured from the origin. This means that

$$
\begin{aligned}
& q_{i}^{+}=\frac{1}{2}\left(1+\left|\alpha_{n-1}\right|+\sqrt{\left(\left|\alpha_{n-1}\right|-1\right)^{2}-4\left|\alpha_{i-1}\right|}\right) \\
& q_{i}^{-}=\frac{1}{2}\left(1+\left|\alpha_{n-1}\right|-\sqrt{\left(\left|\alpha_{n-1}\right|-1\right)^{2}-4\left|\alpha_{i-1}\right|}\right) .
\end{aligned}
$$

Therefore, if $\mu_{i} \geq q_{i}^{+}$, then the largest modulus of any point $z$ in the intersection of the disk centered at the origin with radius $\mu_{i}$ and the region bounded by the curve is given by $\min \left\{\mu_{i}, r_{i}\right\}$ and there is a gap in the values that $|z|$ can attain between $q_{i}^{-}$and $q_{i}^{+}$. This proves statement (2) for $2 \leq i \leq n-2$.

If $\mu_{i}<q_{i}^{+}$, then the largest modulus of $z$ in the intersection is given by $\min \left\{\mu_{i}, q_{i}^{-}\right\}$ and this time there is no gap in the values of $|z|$. This proves statement (3) for $2 \leq i \leq n-2$.

Figure 6] shows a few representative cases when $\left|\alpha_{n-1}\right|>\beta_{i}$. The left and right white dots are the origin and $-\alpha_{n-1}$, respectively. On the left we have a situation where $\mu_{i}<q_{i}^{-}$and on the right $q_{i}^{+}<\mu_{i}<r_{i}$.
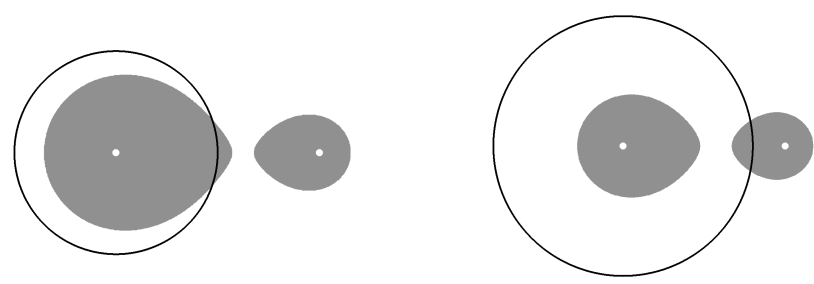

FIGURE 6. Illustration for $\left|\alpha_{n-1}\right|>\beta_{i}$ when $\mu_{i}<q_{i}^{-}$(left) and $\mu_{i}>q_{i}^{+}$(right).

When $i=1$, the oval of Cassini, appearing in $\Phi_{1}$, corresponds to a curve as in Lemma 3.2, obtained by setting $a=-\alpha_{n-1}, b=0, \alpha=0$ and $\beta=\left|\alpha_{0}\right|$ in that lemma. The proof then follows analogously to the proof for $2 \leq i \leq n-2$.

When $i=n-1$, we replace $\Phi_{n-1}$ by $\widetilde{\Phi}_{n-1}$, which we define as

$$
\widetilde{\Phi}_{n-1}=\left\{z \in \mathbb{C}:|z| \leq \mu_{n-1} \text { and }|z|\left|z+\alpha_{n-1}\right| \leq\left|z+\alpha_{n-1}\right|+\left|\alpha_{n-2}\right|\right\} \text {. }
$$


Since $|a+b| \geq|a|-|b|$ for any $a, b \in \mathbb{C}$, we have that $\Phi_{n-1} \subseteq \widetilde{\Phi}_{n-1}$, which implies that we are enlarging the set of possible values of $|z|$. The set of all $z$ satisfying $|z|\left|z+\alpha_{n-1}\right| \leq\left|z+\alpha_{n-1}\right|+\left|\alpha_{n-2}\right|$ is of the same form as the sets appearing in $\Phi_{i}$ for $i=2, \ldots, n-2$ and the proof for $i=n-1$ follows analogously.

When $i=n$, we proceed analogously to the case $i=n-1$ and replace $\Phi_{n}$ by $\widetilde{\Phi}_{n}$, defined as

$$
\widetilde{\Phi}_{n}=\left\{z \in \mathbb{C}:\left|z+\alpha_{n-1}\right| \leq 1 \text { and }|z|\left|z+\alpha_{n-1}\right| \leq 1+\left|\alpha_{n-2}\right|\right\}
$$

We have $\Phi_{n} \subseteq \widetilde{\Phi}_{n}$, and we are therefore once again enlarging the set of possible values of $|z|$. The boundary of the set of all $z$ satisfying $|z|\left|z+\alpha_{n-1}\right| \leq 1+\left|\alpha_{n-2}\right|$ is also an oval of Cassini, but this time the oval is intersected with a disk of radius unity centered at $-\alpha_{n-1}$. We regard the oval as a special case of the curve in Lemma 3.2 with, in the notation of that lemma, $\alpha=0, \beta=1+\left|\alpha_{n-2}\right|, a=-\alpha_{n-1}$, and $b=0$. Furthermore we have

$$
\begin{aligned}
& R_{\text {min }}=\frac{1}{2}\left(-\left|\alpha_{n-1}\right|+\sqrt{\left|\alpha_{n-1}\right|^{2}+4\left(1+\left|\alpha_{n-2}\right|\right)}\right) \\
& R_{\text {max }}=\frac{1}{2}\left(\left|\alpha_{n-1}\right|+\sqrt{\left|\alpha_{n-1}\right|^{2}+4\left(1+\left|\alpha_{n-2}\right|\right)}\right), \\
& Q_{\min }=\frac{1}{2}\left(\left|\alpha_{n-1}\right|-\sqrt{\left|\alpha_{n-1}\right|^{2}-4\left(1+\left|\alpha_{n-2}\right|\right)}\right), \\
& \left.Q_{\max }=\frac{1}{2}\left(\left|\alpha_{n-1}\right|+\sqrt{\left|\alpha_{n-1}\right|^{2}-4\left(1+\left|\alpha_{n-2}\right|\right.}\right)\right)
\end{aligned}
$$

all of which are distances measured from $-\alpha_{n-1}$. If $\left|\alpha_{n-1}\right| \leq \beta_{n}=2 \sqrt{1+\left|\alpha_{n-2}\right|}$, then there is only one loop. When $\left|\alpha_{n-1}\right|>1$, there is a gap from 0 to $\left|\alpha_{n-1}\right|-1$ in the values of $|z|$ for any $z$ in the intersection, while the largest value of $|z|$ is given by the smallest of the numbers $\left|\alpha_{n-1}\right|+1$ and $\left|\alpha_{n-1}\right|+R_{\text {min }}$. There is no gap when $\left|\alpha_{n-1}\right| \leq 1$ and the smallest value for $|z|$ in this case is 0 . Substituting the value of $R_{\min }$ then yields statement (4) of the theorem.

If $\left|\alpha_{n-1}\right|>\beta_{n}=2 \sqrt{1+\left|\alpha_{n-2}\right|}$, there are two loops, one containing $-\alpha_{n-1}$ and one containing the origin. Because here $\left|\alpha_{n-1}\right|>2$, we have that $Q_{\max }>1$, so that the disk centered at $-\alpha_{n-1}$ with radius unity intersects only with the loop containing $-\alpha_{n-1}$. If, in addition, $Q_{\min }>1$, then the loop is not entirely contained in the disk and the smallest and largest values for the modulus of any $z$ in the intersection are $\left|\alpha_{n-1}\right|-1$ (which here is larger than $\left|\alpha_{n-1}\right|-Q_{\min }=q_{n}^{+}$) and $\left|\alpha_{n-1}\right|+\min \left\{1, R_{\min }\right\}$, respectively. This case is illustrated on the left in Figure 7 , where the left and right white dots are the origin and $-\alpha_{n-1}$, respectively. If, on the other hand, $Q_{\min } \leq 1$, then, since $R_{\min } \leq Q_{\min }$, the loop is entirely contained in the closed disk and the smallest and largest values for $|z|$ for any $z$ in the intersection now become $\left|\alpha_{n-1}\right|-Q_{\text {min }}$ (which here is larger than $\left|\alpha_{n-1}\right|-1$ ) and $\left|\alpha_{n-1}\right|+R_{\text {min }}$ (which here is smaller than $\left|\alpha_{n-1}\right|+1$ ), respectively. This situation is illustrated on the right in Figure 7 Substituting the expressions for $Q_{\min }$ and $R_{\min }$, taking into account the definitions of $q_{n}^{+}$and $\mu_{n}$, and combining the two cases $Q_{\min }>1$ and $Q_{\min } \leq 1$ yields statement (5) and concludes the proof of the theorem. 

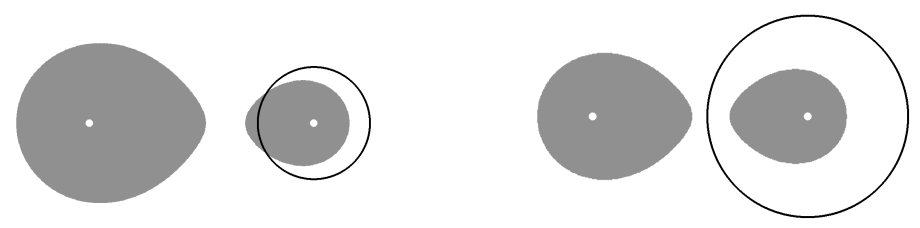

FiguRe 7. Illustration for $\left|\alpha_{n-1}\right|>\beta_{n}$ when $q_{n}^{+}<\left|\alpha_{n-1}\right|-1$ (left) and $q_{n}^{+}>\left|\alpha_{n-1}\right|-1$ (right).

We note that some of the quantities $q_{i}^{-}$and $q_{i}^{+}$defined in the previous theorem may be imaginary, depending on the situation. However, they play no role whenever this occurs. The computation of all the bounds in Theorem 3.3 requires only $\mathcal{O}(n)$ computations. In addition, the bounds for $i=n-1$ and $i=n$ are not the best possible given our inclusion sets since we enlarged the sets $\Phi_{n-1}$ and $\Phi_{n}$ in the interest of obtaining relatively simple explicit expressions for the bounds. They can therefore be improved if so desired at the cost of computing, in general, two intersections of a disk with a quartic. There are only two such intersections to determine, regardless of the degree of the polynomial.

The maximum of the bounds in the previous theorem obviously provides a bound on the modulus of the largest zero. Even after enlarging the sets $\Phi_{n-1}$ and $\Phi_{n}$, this bound can never be worse than the one obtained from the Gershgorin set because of the disks in the definition of the sets $\Phi_{i}(1 \leq i \leq n)$, which are contained in the corresponding Gershgorin disks.

\section{Numerical COMPARISONS}

To illustrate our results, we conclude this paper with some numerical comparisons. The bound on the modulus of the largest zero obtained from the Gershgorin set is

$$
\max \left\{\left|\alpha_{0}\right|, 1+\left|\alpha_{1}\right|, \ldots, 1+\left|\alpha_{n-1}\right|\right\}
$$

which itself is bounded by Cauchy's bound, given by

$$
1+\max \left\{\left|\alpha_{0}\right|,\left|\alpha_{1}\right|, \ldots,\left|\alpha_{n-1}\right|\right\} .
$$

In addition to other classical bounds ([19, pp. 316-319]), there exist several more modern methods, such as in [1], [5], [11, 12], 15, 20], 21], 22., 25], 26], 27, [28, 34, 37, 38, which were already mentioned in the introduction. Judging from the numerical examples in those references, these bounds are, by and large, comparable. Some are better than others, but not uniformly so, and the roles can be reversed, depending on the polynomial. In addition, there are bounds based on a transformation of the polynomial, as in, e.g., 34] and [38, that can be used to improve any bound. It is not our intention to conduct a comprehensive comparison, which would be beyond the scope of this paper. Instead, we have picked a representative method from the aformentioned references, namely the one from [20, to which we will compare our bounds. We have also included a comparison to bounds derived from the Brauer set. We denote the bound from 20. by $b_{J L R}$, the Gershgorin bound by $b_{\Gamma}$, the Brauer bound by $b_{B}$, and the bound based on Theorem 3.3 by $b_{G \Gamma}$.

We generated 1000 monic polynomials of order 50 with random coefficients whose real and complex parts were uniformly distributed on the interval $[-10,10]$, and 
we then computed the ratio of the bound to the modulus of the largest zero for each polynomial; i.e., the closer to one that number is, the better the bound. The average ratio and standard deviation for $b_{\Gamma}$ were 2.0943 and 0.9358 , respectively, and the range of values was $[1.0250,5.9638]$. For $b_{B}$, these numbers were very similar, namely, 2.0484 and 0.9142 , and $[1.0163,5.7734]$, respectively. For $b_{G \Gamma}$ the corresponding results were 1.2522 and 0.2289 , and $[1.0071,2.7216]$, respectively, which is significantly better. For $b_{J L R}$ the corresponding results were 1.2530 and 0.2284 , and $[1.0071,2.7216]$, respectively, which is very similar to what we obtained for $b_{G \Gamma}$, although, as was mentioned before, our bounds can still be improved.

In about $45 \%$ of the cases in our numerical comparisons, Theorem 3.3 also provided an exclusion interval for the moduli of the zeros. Although theoretically possible, Gershgorin's and Brauer's theorem did not produce such intervals. The method from [20] only computes an upper bound on the moduli of the zeros. To illustrate this additional information our bounds can sometimes provide, let us consider $q_{1}(z)=z^{5}+6 z^{4}+4 z^{3}+5 z^{2}+2 i z+3$. The zeros have moduli $0.6113,0.7711,1.0300,1.1364$, and 5.4370 . The bounds $b_{\Gamma}, b_{B}$, and $b_{J L R}$ are 7.000 , 6.8730 , and 6.8541 , respectively, but they provide no more information. However, Theorem 3.3 gives the range $0 \leq|z| \leq 2.3820$ and $4.6180 \leq|z| \leq 6.7417$; i.e., no zeros can have moduli between 2.3820 and 4.6180. Another example is $q_{2}(z)=z^{10}-(15 / 2) z^{9}+5 z^{8}+4 z^{7}-(6+i) z^{6}-2 i z^{5}+2 z^{4}+z^{3}+4 z^{2}+i z+1$. In this case, the zeros have moduli $0.4147,0.6062,0.6668,0.6953,0.9146,0.9459$, $1.0906,1.1649,1.1681$, and 6.6821. From the Gershgorin and Brauer sets, and from [20], we obtain only the upper bounds $b_{\Gamma}, b_{B}$, and $b_{J L R}$ as 8.5, 8.3484, and 8.3299, respectively. On the other hand, Theorem 3.3 yields the range $0 \leq|z| \leq 2.1335$ and $6.3665 \leq|z| \leq 8.2291$; i.e., no zeros can have moduli between 2.1335 and 6.3665 .

We conclude by mentioning that it may be possible to extend our techniques to compute bounds on the real and imaginary parts of polynomial zeros as well. Furthermore, more bounds can be obtained by considering powers of the companion matrix and of its transpose. In addition, as was mentioned in Section 3, our methods can be applied to other types of companion matrices.

\section{REFERENCES}

1. Alpin, Yu.A., Chien, Mao-Ting, and Yeh, Lina. The numerical radius and bounds for zeros of a polynomial. Proc. Amer. Math. Soc., 131 (2003), no. 3, 725-730. MR.1937409(2003h:26021)

2. Barnett, S. Congenial matrices. Linear Algebra Appl., 41 (1981), 277-298. MR815264 (86m:15004)

3. Brauer, A. Limits for the characteristic roots of a matrix. II. Duke Math. J., 14 (1947), 21-26. MR0020540 (8:559i)

4. Bini, D.A., Gemignani, L., and Pan, V.Y. Improved initialization of the accelerated and robust QR-like polynomial root-finding. Electron. Trans. Numer. Anal., 17 (2004), 195-205. MR.2113008 (2006a:65076)

5. Boese, F.G., and Luther, W.J. A note on a classical bound for the moduli of all zeros of a polynomial. IEEE Trans. Automat. Control, 34 (1989), no. 9, 998-1001. MR.1007431 (90h:30015)

6. Calvetti, D., Reichel, L., and Sgallari, F. A modified companion matrix method based on Newton polynomials. Fast algorithms for structured matrices: theory and applications (South Hadley, MA, 2001), 179-186, Contemp. Math., 323, Amer. Math. Soc., Providence, RI, 2003. MR.1999394(2004h:65042)

7. Carstensen, C. Linear construction of companion matrices. Linear Algebra Appl., 149 (1991), 191-214. MR1092878 (92c:15014)

8. Cvetković, L., Kostić, V., and Varga, R.S. A new Geršgorin-type eigenvalue inclusion set. Electron. Trans. Numer. Anal., 18 (2004), 73-80 (electronic). MR2114449 (2005j:65038) 
9. Cvetković, L. and Kostić, V. Between Geršgorin and minimal Geršgorin sets. J. Comput. Appl. Math., 196 (2006), no. 2, 452-458. MR2249436 (2007e:15018)

10. Dashnic, L. S., and Zusmanovich, M. S. O nekotoryh kriteriyah regulyarnosti matric $i$ lokalizacii ih spektra. Zh. Vychisl. Matem. i Matem. Fiz. 5 (1970), 1092-1097.

11. Datt, B., and Govil, N.K. On the location of the zeros of a polynomial. J. Approx. Theory, 24 (1978), no. 1, 78-82. MR510922 (80g:30002)

12. Dehmer, M. On the location of zeros of complex polynomials. J. Inequal. Pure Appl. Math., 7 (2006), Article 26, 13 pp. MR 2217189 (2006m:30011)

13. Edelman, A., and Murakami, H. Polynomial roots from companion matrix eigenvalues. Math. Comp., 64 (1995), 763-776. MR1262279 (95f:65075)

14. Fiedler, M. A note on companion matrices. Linear Algebra Appl., 372 (2003), 325-331. MR 1999154 (2004g:15017)

15. Fujii, Masatoshi, and Kubo, Fumio. Buzano's inequality and bounds for roots of algebraic equations. Proc. Amer. Math. Soc., 117 (1993), no. 2, 359-361. MR1088441 (93d:47014)

16. Gemignani, L. Structured matrix methods for polynomial root-finding. ISSAC 2007, 175-180, ACM, New York, 2007. MR2396200 (2009f:65113)

17. Gerschgorin, S. Über die Abgrenzung der Eigenwerte einer Matrix. Izv. Akad. Nauk SSSR, Ser. Fiz.-Mat., 6 (1931), 749-754.

18. Good, I.J. The colleague matrix, a Chebyshev analogue of the companion matrix. Quart. J. Math. Oxford Ser. (2), 12 (1961), 61-68. MR0132755 (24:A2592)

19. Horn, R.A., and Johnson, C.R. Matrix Analysis. Cambridge University Press, Cambridge, 1988. MR:1084815 (91i:15001)

20. Joyal, A., Labelle, G., and Rahman, Q.I. On the location of zeros of polynomials. Canad. Math. Bull., 10 (1967), 53-63. MR0213513 (35:4374)

21. Kittaneh, F. A numerical radius inequality and an estimate for the numerical radius of the Frobenius companion matrix. Studia Math., 158 (2003), no. 1, 11-17. MR2014548 (2004i:15022)

22. Kittaneh, F. Bounds and a majorization for the real parts of the zeros of polynomials. Proc. Amer. Math. Soc., 135 (2007), 659-664. MR2262860 (2008k:30002)

23. Kolotilina, L.Yu. Generalizations of the Ostrowski-Brauer theorem. Linear Algebra Appl., 364 (2003), 65-80. MR1971087 (2004c:15033)

24. Lawrence, J.D. A Catalog of Special Plane Curves. Dover Publications, Inc., New York, 1972.

25. Linden, H. Bounds for the zeros of polynomials from eigenvalues and singular values of some companion matrices. Linear Algebra Appl., 271 (1998), 41-82. MR.1485162 (98m:65059)

26. Linden, H. Numerical radii of some companion matrices and bounds for the zeros of polynomials. Analytic and geometric inequalities and applications, 205-229, Math. Appl., 478, Kluwer Acad. Publ., Dordrecht, 1999. MR1785871 (2001i:15031)

27. Linden, H. Containment regions for zeros of polynomials from numerical ranges of companion matrices. Linear Algebra Appl., 350 (2002), 125-145. MR1906750 (2003d:30008)

28. Marden, M. Geometry of polynomials. Mathematical Surveys, No. 3, American Mathematical Society, Providence, RI, 1966. MR0225972 (37:1562)

29. Melman, A. Spectral inclusion sets for structured matrices. Linear Algebra Appl., 431 (2009), 633-656. MR2535539

30. Melman, A. An alternative to the Brauer set. Linear Multilinear Algebra, July 2009, DOI:10.1080/03081080902722733, to appear.

31. Neumaier, A. Enclosing clusters of zeros of polynomials. J. Comput. Appl. Math., 156 (2003), 389-401. MR1995854 (2004g:30009)

32. Pan, V.Y. Approximating complex polynomial zeros: Modified Weyl's quadtree construction and improved Newton's iteration. Real computation and complexity (Schloss Dagstuhl, 1998). J. Complexity, 16 (2000), 213-264. MR1762403 (2001g:65063)

33. Peña, J.M. A class of $P$-matrices with applications to the localization of the eigenvalues of a real matrix. SIAM J. Matrix Anal. Appl., 22 (2001), 1027-1037. MR1824055 (2001m:15046)

34. Sun, Yeong-Jeu, and Hsieh, Jer-Guang. A note on the circular bound of polynomial zeros. IEEE Trans. Circuits Syst. I: Fund. Theory Appl., 43 (1996), no. 6, 476-478. MR.1395264 (97a:30004)

35. Varga, R.S. Geršgorin and His Circles. Springer-Verlag, Berlin, 2004. MR2093409 (2005h:15002) 
36. Winkler, J.R. Properties of the companion matrix resultant for Bernstein polynomials. Uncertainty in geometric computations, 185-198, Kluwer Internat. Ser. Engrg. Comput. Sci., 704, Kluwer Acad. Publ., Boston, MA, 2002. MR2063185

37. Zeheb, E. On the largest modulus of polynomial zeros. IEEE Trans. Circuits Syst., 38 (1991), 333-337.

38. Zilović, M.S., Roytman, M., Combettes, P.L., and Swamy, M.N.S. A bound for the zeros of polynomials. IEEE Trans. Circuits Syst. I: Fund. Theory Appl., 38 (1992), no. 3, 476-478.

Department of Applied Mathematics, School of Engineering, Santa Clara UniverSity, Santa Clara, California 95053

E-mail address: amelman@scu.edu 